\title{
Exact Solution and Conservation Laws for Fifth-Order Korteweg-de Vries Equation
}

\author{
Elham M. Al-Ali \\ Mathematics Department, Faculty of Science, University of Tabuk, Tabuk, KSA \\ Email: dr.elham.alali@gmail.com
}

Received September 11, 2013; revised October 11, 2013; accepted October 17, 2013

Copyright (C) 2013 Elham M. Al-Ali et al. This is an open access article distributed under the Creative Commons Attribution License, which permits unrestricted use, distribution, and reproduction in any medium, provided the original work is properly cited.

\begin{abstract}
With the aid of Mathematica, new exact travelling wave solutions for fifth-order KdV equation are obtained by using the solitary wave ansatz method and the $\mathrm{Wu}$ elimination method. The derivation of conservation laws for a fifth-order $\mathrm{KdV}$ equation is considered.
\end{abstract}

Keywords: Soliton Solutions; Conservation Laws; Nonlinear Evolution Equations

\section{Introduction}

It is well-known that nonlinear complex physical phenomena are related to nonlinear partial differential equations (NLPDEs) which are involved in many fields from physics to biology, chemistry, mechanics, etc. As mathematical models of the phenomena, the investigation of exact solutions to the NLPDEs reveals to be very important for the understanding of these physical problems. Many mathematicians and physicists have well understood this importance when the importance of this so they decided to pay special attention to the development of sophisticated methods for constructing exact solutions to the NLPDEs. Thus, a number of powerful methods have been presented.

We can cite the inverse scattering transform [1], the Bäcklund and Darboux transform [2-5], Hirota's bilinear method [6], the homogeneous balance method [7], Jacobi elliptic function method [8], the tanh-method and extended tanh-function method [9-15], F-expansion method [16-18] and so on.

The notion of conservation laws is important in the study of nonlinear evolution equations (NLEEs) appearing in mathematical physics [19]. The mathematical origin of conservation laws results from the formulation of familiar physical laws such as for mass, energy and momentum [20]. As is known, the investigation of conservation laws of the Korteweg-de Vries (KdV) equation led to the discovery of a number of techniques to solve NLEEs [21], e.g., Miura transformation, Lax pair, inverse scattering technique and bi-Hamiltonian structures.
On the other hand, it is useful in the numerical integration of NLEEs [22] (e.g., to control numerical errors); particularly with regard to integrability and linearization, constants of motion, analysis of solutions, and numerical solution methods [23]. Consider a dynamical system,

$$
u_{t}=f\left(x, t, u, u_{x}, u_{t}, u_{x t}, \cdots\right)
$$

where $u=u(x, t)$ is a function of two independent variables $t$ and $x$. The functional $I[u(x, t)]$ is said to be a constant of motion or an integral of Equation (1), if it satisfies $\frac{\mathrm{d}}{\mathrm{d} t} I[u(x, t)]=0$. Generally, we can derive constants of motion from conservation law, which enjoys the general form as [24]

$$
\frac{\mathrm{d}}{\mathrm{d} x} V[u(x, t)]+\frac{\mathrm{d}}{\mathrm{d} t} G[u(x, t)]=0
$$

while the components $V$ and $G$ of the conserved vector $(V, G)$ are functions of $x, t$ and derivatives of $u$. The equality (2) is assumed to be satisfied for any solution of the corresponding system of equations, $V$ is called conserved density and $G$ is called conserved flow. With the assumption that the function $u(x, t)$ and its derivatives with respect to $x$ go to zero sufficiently fast as $|x| \rightarrow \infty$

$$
I[u(x, t)]=\int_{-\infty}^{\infty} V[(x, t)] \mathrm{d} x
$$

is obtained to be a constant of motion. It has already been proved that a large number of NLEEs possess an infinite 
number of conservation laws such as the fifth-order $\mathrm{KdV}$ equation

\section{Exact Solution for Fifth-Order KdV Equation}

With the rapid development of science and technology, the study kernel of modern science is changed from linear to nonlinear step by step. Many nonlinear science problems can simply and exactly be described by using the mathematical model of nonlinear equation. Up to now, many important physical nonlinear evolution equations are found, such as sin-Gordon equation, $\mathrm{KdV}$ equations, Schrodinger equation all possess solitary wave solutions. There exist many methods to seek for the solitary wave solutions, such as inverse scattering method, Hopf-Cole transformation, Miura transformations, Darboux transformation and Bäcklund transformation [2-5], but solving nonlinear equations is still an important task. In this paper, with the aid of Mathematica, a traveling wave solution for a class of fifth-order $\mathrm{KdV}$ equation

$$
u_{t}=u_{5 x}+10 u u_{3 x}+20 u_{x} u_{2 x}+30 u^{2} u_{x}
$$

In order to obtain the soliton solution of (4), the solitary wave ansatz is assumed as

$$
u(x, t)=A \operatorname{sech}^{n} \rho+B, \quad \rho=d(x-c t),
$$

where $A$ is the soliton amplitude, $d$ is the width of the soliton, $C$ is the soliton velocity and $B$ is constant to be determined later, the unknown index $n$ will be determined during the course of derivation of the solution of Equation (4). From Equation (5), I obtain Equation (6).

With the aid of Mathematica or Maple, from (5) and (6), we can get Equation (7).

Now, from Equation (7) equating the exponents $1+3 n$ and $3+2 n$ leads $1+3 n=3+2 n$, which gives $n=2$. From (7) setting the coefficients of $\operatorname{sech}^{3} \rho \sinh \rho, \operatorname{sech}^{5} \rho \sinh \rho$ and $\operatorname{sech}^{7} \rho \sinh \rho$ to zero, I get Equations (8)-(10).

$$
\begin{aligned}
& u^{2}(x, t)=\left(B+A \operatorname{sech}^{n} \rho\right)^{2}, \quad u_{t}=A c d n \operatorname{sech}{ }^{n+1} \rho \sinh \rho . \\
& u_{5 x}=d^{5}\left(-A n^{3} \operatorname{sech}^{1+n} \rho \sinh \rho-4 A n^{2}(1+n) \operatorname{sech}^{1+n} \rho \sinh \rho\right. \\
& -4 A n(1+n)^{2} \operatorname{sech}^{1+n} \rho \sinh \rho-6 A n(1+n)(2+n) \operatorname{sech}^{1+n} \rho \sinh \rho \\
& +A n^{2}(1+n)(2+n) \operatorname{sech}^{3+n} \rho \sinh ^{3} \rho+2 A n(1+n)^{2}(2+n) \operatorname{sech}^{3+n} \rho \sinh ^{3} \rho \\
& +3 A n(1+n)+A n(1+n)(2+n)^{2} \operatorname{sech}^{3+n} \rho \sinh ^{3} \rho \\
& +4 A n(1+n)(2+n)(3+n) \operatorname{sech}^{3+n} \rho \sinh ^{3} \rho \\
& \left.-A n(1+n)(2+n)(3+n)(4+n) \operatorname{sech}^{(5+n)} \rho \sinh ^{5} \rho\right) \\
& u_{3 x}=A n^{2} \operatorname{sech}^{1+n} \rho \sinh \rho+A n(1+n) \operatorname{sech}^{1+n} \rho \sinh \rho \\
& -A n(1+n)(2+n) \operatorname{sech}^{3+n} \rho \sinh ^{3} \rho \\
& u_{x} u_{2 x}=-A^{3} n \operatorname{sech}^{1+n} \rho \sinh \rho-A n \operatorname{sech}^{n} \rho \sinh \rho \\
& +A n(1+n) \operatorname{sech}^{2+n} \rho \sinh ^{2} \rho
\end{aligned}
$$

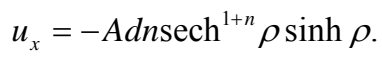

$$
\begin{aligned}
& u_{t}-u_{5 x}-10 u u_{3 x}-20 u_{x} u_{2 x}-30 u^{2} u_{x} \\
& =\left(30 A B^{2} d n+A c d n+10 A B d^{3} n^{3}+A d^{5} n^{5}\right) \operatorname{sech}^{1+n} \rho \sinh \rho \\
& +\left(-20 A B d^{3} n-8 A d^{5} n-30 A B d^{3} n^{2}-20 A d^{5} n^{2}-10 A B d^{3} n^{3}\right. \\
& \left.-20 A d^{5} n^{3}-10 A d^{5} n^{4}-2 A B d^{5} n^{5}\right) \operatorname{sech}^{3+n} \rho \sinh \rho \\
& +\left(24 A d^{5} n+50 A d^{5} n^{2}+35 A d^{5} n^{3}+10 A d^{5} n^{4}+A d^{5} n^{5}\right) \operatorname{sech}^{5+n} \rho \sinh \rho \\
& +\left(60 A^{2} B d n+30 A^{2} d^{3} n^{3}\right) \operatorname{sech}^{1+2 n} \rho \sinh \rho \\
& +\left(-20 A^{2} d^{3} n-50 A^{2} d^{3} n^{2}-30 A^{2} d^{3} n^{3}\right) \operatorname{sech}^{3+2 n} \rho \sinh \rho \\
& +3020 A^{3} d n \operatorname{sech}^{1+3 n} \rho \sinh \rho=0
\end{aligned}
$$




$$
\begin{gathered}
30 B^{2}+c+40 B d^{2}+16 d^{4}=0, \\
-2 B d^{2}+A B+2 A d^{2}-4 d^{4}=0, \\
A^{2}-8 A d^{2}+12 d^{4}=0 .
\end{gathered}
$$

Solving the above system by the aid of $\mathrm{Wu}$ elimination method [25], I obtain the two solutions

and

$$
A=6 d^{2}, B=-2 d^{2}, c=-56 d^{4},
$$

$$
A=2 d^{2}, B=-\frac{4}{3} d^{2}, c=-16 d^{4} .
$$

Then the soliton solutions of the fifth order KdV equation is given by

$$
u_{1}(x, t)=6 d^{2} \operatorname{sech}^{n} d\left(x+56 d^{4} t\right)-2 d^{2},
$$

and

$$
u_{2}(x, t)=2 d^{2} \operatorname{sech}^{n} d\left(x+16 d^{4} t\right)-\frac{4}{3} d^{2}
$$

\section{Systematic Construction Method of Infinitely Many Conservation Laws for Fifth-Order KdV Equation}

We recall the definition $[16,23]$ of a differential equation (DE) that describes a pss. Let $M^{2}$ be a two dimensional differentiable manifold with coordinates $(x, t)$. A DE for a real function $u(x, t)$ describes a pss if it is a necessary and sufficient condition for the existence of differentiable functions

$$
f_{i j}, 1 \leq i \leq 3,1 \leq j \leq 2,
$$

depending on $u$ and its derivatives such that the one-forms

$\omega_{1}=f_{11} \mathrm{~d} x+f_{12} \mathrm{~d} t, \omega_{2}=f_{21} \mathrm{~d} x+f_{22} \mathrm{~d} t, \omega_{3}=f_{31} \mathrm{~d} x+f_{32} \mathrm{~d} t$,

satisfy the structure equations of a pss, i.e.,

$$
\mathrm{d} \omega_{1}=\omega_{3} \wedge \omega_{2}, \mathrm{~d} \omega_{2}=\omega_{1} \wedge \omega_{3}, \mathrm{~d} \omega_{3}=\omega_{1} \wedge \omega_{2} .
$$

As a consequence, each solution of the DE provides a local metric on $M^{2}$, whose Gaussian curvature is constant, equal to -1 . Moreover, the above definition is equivalent to saying that $\mathrm{DE}$ for $u$ is the integrability condition for the problem $[14,26]$ :

$$
\mathrm{d} \phi=\Omega \phi, \quad \phi=\left(\begin{array}{l}
\phi_{1} \\
\phi_{2}
\end{array}\right),
$$

where $\mathrm{d}$ denotes exterior differentiation, $\phi$ is a column vector and the $2 \times 2$ matrix $\Omega\left(\Omega_{i j}, i, j=1,2\right)$ is traceless

$$
\Omega=\frac{1}{2}\left(\begin{array}{cc}
\omega_{2} & \omega_{1}-\omega_{3} \\
\omega_{1}+\omega_{3} & -\omega_{2}
\end{array}\right) .
$$

Take

$$
\Omega=\left(\begin{array}{cc}
\eta \mathrm{d} x+A \mathrm{~d} t & q \mathrm{~d} x+B \mathrm{~d} t \\
r \mathrm{~d} x+C \mathrm{~d} t & -\eta \mathrm{d} x-A \mathrm{~d} t
\end{array}\right)=S \mathrm{~d} x+T \mathrm{~d} t,
$$

from Equations (18) and (19), we obtain

$$
\phi_{x}=S \phi, \quad \phi_{t}=T \phi,
$$

where $S$ and $T$ are two $2 \times 2$ null-trace matrices

$$
\begin{aligned}
& S=\left(\begin{array}{cc}
\eta & q \\
r & -\eta
\end{array}\right), \\
& T=\left(\begin{array}{cc}
A & B \\
C & -A
\end{array}\right) .
\end{aligned}
$$

Here $\eta$ is a parameter, independent of $x$ and $t$, while $q$ and $r$ are functions of $x$ and $t$. Now

$$
0=\mathrm{d}^{2} \phi=\mathrm{d} \Omega \phi-\Omega \wedge \mathrm{d} \phi=(\mathrm{d} \Omega-\Omega \wedge \Omega) \phi,
$$

which requires the vanishing of the two form

$$
\Theta \equiv \mathrm{d} \Omega-\Omega \wedge \Omega=0,
$$

or in component form

$$
\begin{aligned}
& -A_{x}+q C-r B=0, \\
& q_{t}-2 A q-B_{x}+2 \eta B=0, \\
& r_{t}-C_{x}+2 A r-2 \eta C=0,
\end{aligned}
$$

or

$$
\begin{aligned}
& -f_{11, t}+f_{12, x}=f_{31} f_{22}-f_{21} f_{32,} \\
& -f_{21, t}+f_{22, x}=f_{11} f_{32}-f_{12} f_{31,} \\
& -f_{31, t}+f_{32, x}=f_{11} f_{22}-f_{12} f_{21,}
\end{aligned}
$$

where

$$
\begin{aligned}
& q=\frac{1}{2}\left(f_{11}-f_{31}\right), r=\frac{1}{2}\left(f_{11}+f_{31}\right) \\
& A=\frac{1}{2} f_{22}, B=\frac{1}{2}\left(f_{12}-f_{32}\right), c=\frac{1}{2}\left(f_{12}+f_{32}\right) .
\end{aligned}
$$

Chern and Tenenblat [27] obtained Equation (24) directly from the structure equations (17). By suitably choosing $r, A, B$ and $C$ in (24), we shall obtain various fifth order $\mathrm{KdV}$ equation which $q$ must satisfy. Konno and Wadati introduced the function [28]

$$
\Gamma=\frac{\phi_{1}}{\phi_{2}},
$$

this function first appeared used and explained in the geometric context of pseudo spherical equations in $[11,13]$, and see also the classical papers by Sasaki [29] and Chern-Tenenblat [27]. Then Equation (20) is reduced to the Riccati equations:

$$
\begin{array}{r}
\frac{\partial \Gamma}{\partial x}=\eta \Gamma-r \Gamma^{2}+q, \\
\frac{\partial \Gamma}{\partial t}=2 A \Gamma-C \Gamma^{2}+B .
\end{array}
$$

Equations (28) and (29) imply that 


$$
c \Gamma_{x}-r \Gamma_{t}=(c q-r B)+(\eta c-2 A r) \Gamma .
$$

to both sides and using the expression $\left(-r \Gamma_{t}\right)$ Adding $A_{x}=q_{c}-r$ from (24), Equation (30) takes the form

$$
\frac{\partial}{\partial t}(r \Gamma)+\frac{\partial}{\partial x}(A-c \Gamma)=0,
$$

let us show how an infinite number of conservation laws result from these results. The Riccati equations for $\Gamma$ in the $x$-variable can be rearranged to take the form

$$
\eta(r \Gamma)=-r q+(r \Gamma)^{2}+r \frac{\partial}{\partial x}\left(\frac{r \Gamma}{r}\right)
$$

A similar pair of equations can be obtained for the $t$ derivatives. Expand $r \Gamma$ into a power series in the inverse of $\eta$ so that

$$
r \Gamma(x, t, \eta)=\sum_{n=1}^{\infty} \varnothing_{n}(x, t) \eta^{-n}
$$

the $\varnothing_{n}$ are unknown at this point, however a recursion relation can be obtained for the $\varnothing_{n}$ by using (32), substituting (33) into the $\Gamma$ equation in (32), I find that

$$
\begin{aligned}
& \sum_{n=1}^{\infty} \varnothing_{n}(x, t) \eta^{-n+1} \\
& =-r q+\left(\sum_{n=1}^{\infty} \varnothing_{n}(x, t) \eta^{-n}\right)^{2} \\
& \quad+r\left[\sum_{n=1}^{\infty}\left(\frac{\varnothing_{n}(x, t)}{r}\right) \eta^{-n}\right]
\end{aligned}
$$

Appling the Cauchy product formula

$$
\left(\sum_{n=1}^{\infty} \varnothing_{n}(x, t) \eta^{-n}\right)^{2}=\sum_{n=2}^{\infty}\left(\sum_{j}^{n-1} \varnothing_{j} \varnothing_{n-j}\right) \eta^{-n}
$$

in (34), then I obtain

$$
\begin{aligned}
\varnothing_{1} & +\varnothing_{2} \eta^{-1}+\sum_{n=2}^{\infty} \varnothing_{n+1}(x, t) \eta^{-n} \\
= & -r q+\sum_{n=2}^{\infty}\left(\sum_{j}^{n-1} \varnothing_{j} \varnothing_{n-j}(x, t)\right) \eta^{-n} \\
& +r\left(\frac{\varnothing_{1}(x, t)}{r}\right) \eta^{-1}+r\left[\sum_{n=2}^{\infty}\left(\frac{\varnothing_{n}(x, t)}{r}\right) \eta^{-n}\right] .
\end{aligned}
$$

Now equate powers of $\eta$ on both sides of this expression to produce the set of recursions,

$$
\begin{aligned}
& \varnothing_{1}=-r q, \quad \varnothing_{2}=-r q_{x} \\
& \varnothing_{n+1}=r\left(\frac{\varnothing_{n}}{r}\right)_{x}+\sum_{k=1}^{n-1} \varnothing_{k}(x, t) \varnothing_{n-k}(x, t) . \quad n \geq 2
\end{aligned}
$$

Substituting (33) into (31), the following system of conservation laws appears

$$
\sum_{n=1}^{\infty} \frac{\partial \varnothing_{n}(x, t)}{\partial t} \eta^{-n}=\frac{\partial}{\partial x}\left[-A+c \sum_{n=1}^{\infty} \frac{\varnothing_{n}(x, t)}{r} \eta^{-n}\right] \text {. }
$$

This procedure generates an infinite number of conservation laws for the equation under examination. To obtain conservation laws using (37) in a particular example using this procedure, let us consider the fifth-order
$\mathrm{KdV}$ Equation (4), for Equation (4)

$$
\begin{aligned}
q= & 1, r=-u, A=-u_{3 x}+\eta u_{2 x}-6 u u_{x} \\
& -\eta^{2} u_{x}+3 \eta u^{2}+\eta^{3} u+\frac{\eta^{5}}{2}, \\
B= & 2 u_{2 x}+\eta^{4}+6 u^{2}+2 \eta^{2} u, \\
c= & -u_{4 x}+\eta u_{3 x}-8 u u_{2 x}-\eta^{2} u_{2 x}-6 u_{x}^{2} \\
& +6 \eta u u_{x}+\eta^{3} u_{x}-6 u^{3}-2 \eta^{2} u^{2}-\eta^{4} u .
\end{aligned}
$$

Substituting (38) into (24), I obtain the fifth-order $\mathrm{KdV}$ Equation (4). Putting (38) into (37), it is found that

$$
\sum_{n=1}^{\infty} \frac{\partial \varnothing_{n}(x, t)}{\partial t} \eta^{-n}=\frac{\partial}{\partial x}\left[-A+c \sum_{n=1}^{\infty} \frac{\varnothing_{n}(x, t)}{r} \eta^{-n}\right]
$$

Since

$$
\varnothing_{1}=u, \varnothing_{2}=0, \varnothing_{3}=u^{2}, \varnothing_{4}=u u_{x}, \cdots
$$

\section{Conclusions}

A soliton is a localized pulse-like nonlinear wave that possesses remarkable stability properties. Typically, problems that admit soliton solutions are in the form of evolution equations that describe how some variable or set of variables evolve in time from a given state. The equations may take a variety of forms, for example, PDEs, differential difference equations, partial difference equations, and integro-differential equations, as well as coupled ODEs of finite order.

In this paper, we considered the construction of exact solutions to fifth-order KdV equation. We obtain travelling wave solutions for the above equation by using the solitary wave ansatz method with the aid of Mathematica.

The soliton phenomena and conservation laws of NLEEs represent an important and well established field of modern physics, mathematical physics and applied mathematics. Solitons are found in various areas of physics from hydrodynamics and plasma physics, nonlinear optics and solid state physics, to field theory and gravitation. NLEEs which describe soliton phenomena have a universal character.

A travelling wave of permanent form has already been met; this is the solitary wave solution of the NLEE itself. Such a wave is a special solution of the governing equation which does not change its shape and which propagates at constant speed.

The Soliton equations play a central role in the field of integrable systems and also play a fundamental role in several other areas of mathematics and physics.

\section{REFERENCES}

[1] M. J. Abolwitz and P. A. Clarkson, "Solitons, Nonlinear Evolution Equation and Inverse Scattering," Cambridge University Press, Cambridge, 1991. http://dx.doi.org/10.1017/CBO9780511623998 
[2] C. Rogers and P. Wong, "On reciprocal B€ a Acklund Transformations of Inverse Scattering Schemes," Physica Scripta, Vol. 30, 1984, pp. 10-14. http://dx.doi.org/10.1088/0031-8949/30/1/003

[3] A. H. Khater, D. K. Callebaut, A. A. Abdalla, A. R. Shehata and S. M. Sayed, "Backlund Transformations and Exact Solutions for Self-Dual SU(3) Yang-Mills Equations," IL Nuovo Cimento B, Vol. 114, 1999, pp. 1-10.

[4] C. Qu, Y. Si and R. Liu, "On Affine Sawada-Kotera Equation," Chaos, Solitons \& Fractals, Vol. 15, No. 1, 2003, pp. 131-139.

http://dx.doi.org/10.1016/S0960-0779(02)00121-2

[5] O. C. Wright, "The Darboux Transformation of Some Manakov Systems," Applied Mathematics Letters, Vol. 16, No. 5, 2003, pp. 647-652.

http://dx.doi.org/10.1016/S0893-9659(03)00061-2

[6] R. Hirota, "The Direct Method in Soliton Theory," Cambridge University Press, Cambridge, 2004.

[7] A. H. Khater, D. K. Callebaut and S. M. Sayed, "Exact Solutions for Some Nonlinear Evolution Equations which Describe Pseudospherical Surfaces," Journal of Computational and Applied Mathematics, Vol. 189, No. 1-2, 2006, pp. 387-411. http://dx.doi.org/10.1016/j.cam.2005.10.007

[8] S. K. Liu, Z. T. Fu and S. D. Liu, "Jacobi Elliptic Function Expansion Method and Periodic Wave Solutions of Nonlinear Wave Equations," Physics Letters A, Vol. 289, No. 1-2, 2001, pp. 69-74. http://dx.doi.org/10.1016/S0375-9601(01)00580-1

[9] E. Fan, "Extended Tanh-Function Method and Its Applications to Nonlinear Equations," Physics Letters A, Vol. 277, 2000, pp. 212-219. http://dx.doi.org/10.1016/S0375-9601(00)00725-8

[10] W. Malfliet and W. Hereman, "The Tanh Method I. Exact Solutions of Nonlinear Wave Equations," Physica Scripta, Vol. 54, No. 6, 1996, pp. 569-575. http://dx.doi.org/10.1088/0031-8949/54/6/004

[11] K. Chadan and P. C. Sabatier, "Inverse Problem in Quantum Scattering Theory," Springer, New York, 1977. http://dx.doi.org/10.1007/978-3-662-12125-2

[12] M. J. Ablowitz, S. Chakravarty and R. Halburd, "On Painlevé and Darboux-Halphen Type Equations, in the Painlevé Property, One Century Later," In: R. Conte, Ed., CRM Series in Mathematical Physics, Springer, Berlin, 1998.

[13] M. Elham Al-Ali, "Traveling Wave Solutions for a Generalized Kawahara and Hunter-Saxton Equations," International Journal of Mathematical Analysis, Vol. 7, 2013, pp. 1647-1666.

[14] S. M. Sayed, "The Bäcklund Transformations, Exact Solutions, and Conservation Laws for the Compound Modified Korteweg-de Vries-Sine-Gordon Equations which describe Pseudospherical Surfaces," Journal of Applied Mathematics, Vol. 2013, 2013, pp. 1-7. http://dx.doi.org/10.1155/2013/613065

[15] V. B. Matveev and M. A. Salle, "Darboux Transformations and Solitons," Springer-Verlag, Berlin, 1991. http://dx.doi.org/10.1007/978-3-662-00922-2
[16] K. Tenenblat, "Transformations of Manifolds and Applications to Deferential Equations, Pitman Monographs and Surveys in Pure and Applied Mathematics 93," Addison Wesley Longman, England, 1998.

[17] A. M. Wazwaz, "New Compactons, Solitons and Periodic Solutions for Nonlinear Variants of the KdV and the KP Equations," Chaos, Solitons \& Fractals, Vol. 22, 2004, pp. 249-260. http://dx.doi.org/10.1016/j.chaos.2004.01.005

[18] A. M. Wazwaz, "Two Reliable Methods for Solving Variants of the KdV Equation with Compact and Noncompact Structures," Chaos, Solitons \& Fractals, Vol. 28, No. 2, 2006, pp. 454-462. http://dx.doi.org/10.1016/j.chaos.2005.06.004

[19] X. G. Geng and H. Wang, "Coupled Camassa-Holm Equations, $N$-Peakons and Infinitely Many Conservation Laws," Journal of Mathematical Analysis and Applications, Vol. 403, 2013, pp. 262-271.

http://dx.doi.org/10.1016/j.jmaa.2013.02.030

[20] A. H. Khater, D. K. Callebaut and S. M. Sayed, "Conservation Laws for Some Nonlinear Evolution Equations which Describe Pseudo-Spherical Surfaces," Journal of Geometry and Physics, Vol. 51, No. 3, 2004, pp. 332-352. http://dx.doi.org/10.1016/j.geomphys.2003.11.009

[21] J. A. Cavalcante and K. Tenenblat, "Conservation Laws for Nonlinear Evolution Equations," Journal of Mathematical Physics, Vol. 29, 1988, pp. 1044-1059. http://dx.doi.org/10.1063/1.528020

[22] R. Beals, M. Rabelo and K. Tenenblat, "Backlund Transformations and Inverse Scattering Solutions for Some Pseudo-Spherical Surfaces," Studies in Applied Mathematics, Vol. 81, 1989, pp. 125-134.

[23] E. G. Reyes, "Conservation Laws and Calapso-Guichard Deformations of Equations Describing Pseudo-Spherical Surfaces," Journal of Mathematical Physics, Vol. 41, 2000, pp. 2968-2979. http://dx.doi.org/10.1063/1.533284

[24] E. G. Reyes, "On Geometrically Integrable Equations and Hierarchies of Pseudo-Spherical Type," Contemporary Mathematics, Vol. 285, 2001, pp. 145-156. http://dx.doi.org/10.1090/conm/285/04740

[25] W. T. Wu, "Polynomial Equations-Solving and Its Applications," Algorithms and Computation, Beijing, 1994, pp. 1-9.

[26] M. J. Ablowitz, D. J. Kaup, A. C. Newell and H. Segur, "The Inverse Scattering Transform-Fourier Analysis for Nonlinear Problems," Studies in Applied Mathematics, Vol. 53, 1974, pp. 249-257.

[27] S. S. Chern and K. Tenenblat, "Pseudospherical Surfaces and Evolution Equations," Studies in Applied Mathematics, Vol. 74, 1986, pp. 55-83.

[28] K. Konno and M. Wadati, "Simple Derivation of Backlund Transformation from Riccati Form of Inverse Method," Progress of Theoretical Physics, Vol. 53, 1975, pp. 1652-1656. http://dx.doi.org/10.1143/PTP.53.1652

[29] R. Sasaki, "Soliton Equations and Pseudospherical Surfaces," Nuclear Physics B, Vol. 154, 1979, pp. 343-357. http://dx.doi.org/10.1016/0550-3213(79)90517-0 